\title{
Aplicación de técnicas de balanceo de línea para equilibrar las cargas de trabajo en el área de almacenaje de una bodega de almacenamiento
}

\author{
Application of line balancing techniques to balance workloads in the storage area of a \\ warehouse storage.
}

\author{
Diego León Peña Orozco, Ángela María Neira García, Reynel Alberto Ruiz Grisales \\ Ingeniero Industrial, Universidad del Valle, Guadalajara de Buga, Colombia \\ Correo-e: diego.l.pena@correounivalle.edu.co,
}

\begin{abstract}
Resumen- Hoy en día el manejo de las bodegas de almacenamiento de una empresa industrial se ha convertido en centros enfocados al servicio, donde el personal por lo general realiza tareas como: recibir productos de clientes internos, inspeccionar y clasificar, recoger, almacenar y preparar los productos para su despacho y posteriormente enviarlos a los clientes o consumidor final. En este sentido una de las problemáticas más frecuentes es la de asegurar un balance óptimo de la línea para que el flujo sea continuo y uniforme, buscando aumentar la velocidad del proceso, aumentar la productividad, disminuir tiempos de espera y costos operativos.
\end{abstract}

Palabras clave - Balanceo de línea, eficiencia, flow shop, sistema productivo, tasa de producción, tiempo de ciclo.

Abstract - Today, the management of the storage holds an industrial company have become focused on service centers, where the staff usually perform tasks such as receiving products from internal clients, inspection and classification, picking, storage and deliveries to the final customers. The most frequently problem is to get an optimal balance of the line increasing the speed of the process, productivity and reducing dead times and operating costs.
Key Word - balancing line, cycle time, efficiency, flow shop, production rate, production system.

\section{INTRODUCCIÓN}

Un sistema productivo ya sea del tipo flow shop, job shop, por proyectos o manufactura modular, está enfocado a mejorar su eficiencia elevando su productividad. Por incremento en la productividad se entiende, el aumento en la producción por hora de trabajo, mediante el uso de técnicas como métodos de trabajo, estudio de tiempos y sistemas de compensación.

El presente trabajo tiene como finalidad desarrollar un estudio de tiempos, en el área de almacenaje de una bodega de almacenamiento en una empresa industrial típica del Valle del Cauca, cuya operación se puede concebir como un sistema flow shop, para mejorar el desempeño en las actividades de recolección, almacenamiento, preparación y alistamiento del producto, mediante la aplicación de técnicas de balanceo de línea. 
En este documento se estudiarán los conceptos tales como estudio de tiempos, balanceo de línea, tiempo de ciclo, entre otros, los cuales permitirán abordar este problema.

\section{REVISIÓN DE LA LITERATURA}

Los métodos de estudio de tiempos fueron originalmente desarrollados por Taylor y continúan siendo la técnica más ampliamente utilizada para medir el trabajo, que consta de tareas breves y repetitivas. La tarea correspondiente se descompone en movimientos básicos y cada elemento es medido con un cronómetro. En seguida, el tiempo promedio de varios ciclos es calculado y ajustado para la velocidad y habilidad o tasa de ejecución (TE) del trabajador que es objeto del estudio. Finalmente, se aplica un factor de concesión (FC) para necesidades personales, retrasos inevitables y fatiga [4]. Las siguientes definiciones son importantes conocerlas para comprender mejor en qué consiste el estudio de tiempos:

- Estudio de tiempos: esta actividad implica la técnica de establecer un estándar de tiempo permisible para realizar una tarea determinada, con base en la medición del contenido de trabajo del método prescrito, con la debida consideración de la fatiga, las demoras personales y los retrasos inevitables. Un analista de estudio de tiempos tiene varias técnicas que se utilizan para establecer un estándar: el estudio cronométrico de tiempos, datos estándares, datos de los movimientos fundamentales, muestreo del trabajo y estimaciones basadas en datos históricos.

- El tiempo estándar de una operación: es el tiempo que debería tardarse un operario calificado en realizar una operación, utilizando un método definido, a una velocidad normal y trabajando en condiciones normales de operación (iluminación, ventilación, ambiente).

Muchas veces nos preguntamos por qué un operario no cumple su estándar, surgiendo interrogantes como, por ejemplo:

a. ¿Es un operario calificado, o le falta experiencia?

b. ¿Está utilizando el método correcto?

c. ¿Está trabajando a una velocidad normal?

d. ¿Las condiciones de trabajo (iluminación, ventilación, ruido...) son aceptables?

A través del tiempo varios autores han estudiado y desarrollado diversos modelos y métodos, algunos heurísticos, otros matemáticos, para encontrar el equilibrio de las cargas de trabajo y asegurar el flujo continuo de los productos. Uno de ellos es el balanceo de línea.

Balanceo de Línea [1]: el balance de líneas es un factor crítico para la productividad de una empresa, su objetivo es hallar una distribución de la capacidad adecuada, para asegurar un flujo continuo y uniforme de los productos, a través de los diferentes procesos dentro de la planta, encontrando las formas para igualar los tiempos de trabajo en todas las estaciones, para maximizar aprovechamiento posible de la mano de obra y del equipo, y de ese modo reducir o eliminar el tiempo ocioso. Deben existir ciertas condiciones para que la producción en línea sea práctica:

$\checkmark$ Cantidad: el volumen o cantidad de producción debe ser suficiente para cubrir el costo de la preparación de la línea, esto depende del ritmo de producción y de la duración que tendrá la tarea.

$\checkmark$ Equilibrio: los tiempos necesarios para cada operación en línea deben ser aproximadamente iguales.

$\checkmark$ Continuidad: deben tomarse precauciones para asegurar un aprovisionamiento continuo del material, piezas, subensambles y la prevención de fallas de equipo.

Dos aspectos importantes en el balanceo de línea de ensamble, son la tasa de producción y la eficiencia. Por definición se tiene que la tasa de producción es la cantidad de artículos o servicios que se realizan en cierta cantidad de tiempo, y la eficiencia está vinculada a utilizar los medios disponibles de manera racional para alcanzar un objetivo fijado con anterioridad en el menor tiempo posible y con el mínimo uso posible de los recursos, lo que supone una optimización.

Para calcular la eficiencia del balanceo de una línea se divide el tiempo total de las tareas entre el producto del número de estaciones de trabajo requeridas, por el tiempo de ciclo asignado [6].

$$
E=\frac{\sum t_{\text {tareas }}}{(\text { No.real ET }) *\left(T C_{\text {asignado }}\right)}
$$

Donde:

$\checkmark$ Tiempos de tareas $\left(\boldsymbol{t}_{\text {tareas }}\right)$ : es el tiempo que lleva hacer el producto.

$\checkmark$ Tiempo de ciclo (TC): es el intervalo que transcurre para que los productos terminados dejen la línea operativa o de producción. Si el tiempo requerido en cualquier estación 
excede el disponible para un trabajador, tienen que agregarse trabajadores.

$$
T C=\frac{t \text { produc. disponible por día }}{\text { Unid.requeridas por día }}
$$

$\checkmark$ El número mínimo teórico de trabajo (ET = estación de trabajo): es el tiempo total de duración de las tareas, dividido entre el tiempo del ciclo.

$$
\text { No.mín ET }=\sum_{i=1}^{n} \frac{t \text { para tarea } i}{T C}
$$

Existen varias clasificaciones de los problemas de balanceo de línea, las más conocidas son las propuestas por Baybars en las que se distinguen dos tipos de problemas clásicos: Simple (Simple Assembly Line Balancing Problem, SALBP) o General (General Assembly Line Balancing Problem, GALBP).

\section{SALBP: Simple Assembly Line Balancing Problem}

Los SALBP propuestos principalmente por Baybars (1986) y Scholl (1999), engloban los problemas de equilibrado más simples, presupone que todos los parámetros se conocen con certeza, así, una tarea no puede ser dividida entre dos o más estaciones de trabajo, existen secuencias tecnológicas que deben respetarse (que determinan relaciones de precedencia e incompatibilidad entre las tareas) y todas las tareas deben llevarse a cabo. También se presupone que todas las estaciones de trabajo están equipadas para poder realizar cualquier tarea y bajo el mismo costo. A la vez, la duración de realización de una tarea es independiente de la estación de trabajo a la que sea asignada y de las tareas que la hayan precedido en ella.

Teniendo en cuenta las anteriores características comunes, se pueden distinguir cuatro casos distintos de SALBP:

a) SALBP-1: se caracteriza por disponer de un tiempo de ciclo asignado (o tasa de producción) y desea minimizar el número de estaciones que se requiere para llevar a cabo el proceso. Es muy común que se dé cuando la demanda externa puede ser estimada y un nuevo sistema de montaje vaya a ser instalado.

b) SALBP-2: a diferencia del caso anterior, se parte de un número de estaciones fijado y se desea minimizar el tiempo de ciclo de la línea de ensamble en cuestión. Se acostumbra a presentar dicho problema cuando la línea de ensamble ya existe.

c) SALBP-E: se busca maximizar la eficiencia de la línea de ensamble, o lo que es equivalente, se desea minimizar el producto del número de estaciones y el tiempo de ciclo.

d) SALBP-F: en este caso no se busca maximizar ni minimizar ningún valor, sino que se limita a encontrar, si existe, una solución factible para una combinación cualquiera de tiempo de ciclo y número de estaciones.

\section{GALBP: General Assembly Line Balancing Problem}

Engloba todos aquellos problemas de equilibrio de líneas de ensamble que no son SALBP, los más comunes son los que tienen, por ejemplo: modelos mixtos, estaciones en paralelo, procesamientos alternativos, tiempos de proceso variables. Se caracterizan por dar cabida a problemas más reales y cotidianos que los SALBP. Se destacan cuatro tipos diferentes de problemas GALBP:

a) UALBP (U-line Assembly Line Balancing Problem): se caracterizan por que en vez de tratar con una línea en serie, trabaja con una tipo U. En una línea tipo U las estaciones pueden ser colocadas de tal manera que se pueden manejar a la vez dos piezas en diferentes posiciones de la línea. Como se puede consultar en diferentes ejemplos, en esta tipología de línea hay un mayor número de posibilidades de asignar las tareas a las estaciones, con lo que el problema se puede resolver de manera más eficiente que cuando se tiene una línea en serie. También se pueden distinguir: UALBP1 (minimizar el número de estaciones), UALBP-2 (minimizar el tiempo de ciclo) y UALBP-E (maximizar la eficiencia de la línea).

b) MALBP (Mixed-Model Assembly Line Balancing Problem): el equilibrado de líneas con modelos mixtos se caracterizan por tener varios modelos de un mismo producto, teniendo un conjunto de tareas común a realizar en la totalidad de los modelos y sin tener en cuenta tiempos de preparación de la línea. Al igual que en las tipologías anteriores, también se presentan problemas MALBP-1, MALBP-2 y MALBP-E.

c) RALBP (Robotic Assembly Line Balancing Problem): en el equilibrado de líneas robotizadas se desea optimizar la ejecución de las tareas en la línea, considerando tanto la asignación de las tareas a cada una de las estaciones como 
la destinación de cada uno de los robots a las diferentes estaciones.

d) MOALBP (Multi-Objective Assembly Line Balancing Problem): equilibrado de líneas con objetivos múltiples se caracterizan por buscar varios objetivos a la vez combinando, por ejemplo, minimizar el número de estaciones, el costo o el número de trabajadores, y maximizar la eficiencia. En la mayoría de los problemas de equilibrado de líneas se buscan cumplir objetivos múltiples.

Los dos tipos de problemas (SALBP y GALBP), se pueden subdividir teniendo en cuenta la clasificación dada por Ghosh y Gagnon (1989):

- El tipo de producto que se procesa en la línea: modelo simple (SM) y modelo mixto o múltiple (MM)

- La variabilidad de las duraciones de las tareas: determinista (D) o estocástico (S).

\begin{tabular}{|c|c|c|}
\hline $\begin{array}{c}\text { Tipos de } \\
\text { problemas } \\
\text { ALB. }\end{array}$ & \multicolumn{2}{|c|}{$\begin{array}{c}\text { Tipo de producto } \\
\text { ensamblado }\end{array}$} \\
\hline $\begin{array}{c}\text { Tiempo de la } \\
\text { tarea }\end{array}$ & Simple & Mixto/múltiple \\
\hline \multirow{2}{*}{$\begin{array}{c}\text { Determinístico } \\
\text { Estocástico }\end{array}$} & $\begin{array}{c}\text { SALBP- } \\
\text { SMD }\end{array}$ & SALBP-MMD \\
\cline { 2 - 3 } & $\begin{array}{c}\text { GALBP- } \\
\text { SMD }\end{array}$ & GALBP-MMD \\
& SALBP- & SALBP-MMS \\
\cline { 2 - 3 } & GALBP- & GALBP-MMS \\
\hline & SMS & \\
\hline
\end{tabular}

Tabla 1. Tipos de problemas de ALB.

En la literatura, destacan autores como D. J. Fonseca, C. L. Guest, M. Elam y C. L. Karr, quienes en su trabajo fundamentan los inicios de las líneas de ensamble, los problemas del balanceo y las limitaciones para que las empresas apliquen los métodos analíticos en sus líneas de producción. En este artículo se menciona que las líneas de ensamble de manufactura fueron diseñadas para ser eficientes $\mathrm{y}$ altamente productivas para la manufactura de un producto en particular.

Salveson (1955), publicó el primer artículo sobre el problema de balanceo de la línea de ensamble y desde entonces, muchas investigaciones y ampliaciones se han realizado sobre el problema original. Gutjahar y Nemhauster (1964) mostraron que el problema de ALBP cae dentro de la clase NP-Hard de problemas de optimización combinatoria. Esto significa que una solución óptima no está garantizada para problemas de tamaño significativo. Así, los métodos heurísticos se han convertido en las técnicas más populares para resolver el problema [5].

Becker y Scholl (2006) investigaron los problemas generalizados del equilibrado de líneas de ensamble con énfasis en las características específicas, tales como en las funciones de costos, selección de equipos y de diseño, ya sea en paralelo, en forma de $U$ y línea de producción de modelo mixto. Más recientemente, Boysen (2007) profundamente investigó los problemas de equilibrio de la línea de ensamble y destacó algunas extensiones de modelos pertinentes. La primera investigación no incluye específicamente configuraciones dependientes de la secuencia entre las tareas; sin embargo, en la segunda investigación se menciona este concepto como un área para la investigación futura, que elabora sobre la falta de atención hacia este tema.

Otro gran personaje que aportó grandes investigaciones a este tipo de problemas en la línea de ensamble es Scholl (2008), quien propuso un problema que se refirió a la dificultad de la secuencia dependiente de los problemas de balanceo de la línea de ensamble (SALBP) que hacen referencia a que cada vez que una tarea j se realiza después de que la tarea i se ha terminado, su estándar de tiempo t se incrementa [10].

Ahora bien para aproximarse de una manera más cercana a la problemática analizada en este trabajo, se han estudiado artículos de Pedro Daniel Medina, Jorge Hernán Restrepo, Eduardo Arturo Cruz y Carlos Saracho, como: "Una heurística de balanceo de línea de producción aplicada a una malla curricular" (2006) con el objetivo de aplicar la heurística de optimización de Helgeson \& Birnie para efectuar el posterior balanceo de asignaturas por semestre dentro de la malla curricular; y "Problemas de balanceo de línea SALBP-1 y SALBP-2: Un caso de estudio" (2008) donde se plantean modelos matemáticos para los dos tipos de problema simple, mediante el algoritmo COMSOAL, el cual permite encontrar varias soluciones posibles. 
Otro caso de estudio desarrollado por los mismos autores es "Balanceo de un módulo de confección utilizando el algoritmo de Helgeson \& Birnie", explica que en la elaboración de una prenda de vestir se presenta un conjunto de actividades que están restringidas en precedencia, su naturaleza discreta posibilita que se puedan agrupar de varias maneras dentro de un proceso de producción. El determinar la configuración óptima que minimice el tiempo ocioso total es una tarea difícil e implica utilizar técnicas que permitan llegar a dicho resultado. El problema puede estar limitado por un número de estaciones o una tasa de producción predeterminada, por tanto, es importante definir una metodología que permita alcanzar los resultados que logran el mejor equilibrio en la asignación de carga de trabajo en el módulo [9].

Uno de los problemas que generalmente se presentan dentro de un proceso manufacturero, como lo es el de la confección, es el de asegurar un flujo continuo y uniforme de los productos, en sus diferentes etapas de elaboración, a través de los diversos procesos dentro de la planta o módulo de confección [7]. Lo anterior se ve afectado por muchas variables, como los tiempos de operación por parte de las personas, que a su vez, dependen de otros factores, tales como el cansancio, la curva de rendimiento, el nivel de aprendizaje, la dificultad de la operación, la temperatura, etc. Además de la mano de obra, se cuenta con recursos que se pueden ver limitados en un momento dado: las máquinas, los materiales, los insumos, etc.

Aunque el problema de balanceo de la línea de ensamble ha recibido atención significativa, muchas compañías aun no utilizan los métodos propuestos en la literatura. Esta realidad puede ser vista en una investigación conducida por Chase en 1974, que reporto que aproximadamente solo el $5 \%$ de las compañías con líneas de producción utilizan las técnicas de balanceo de líneas tradicionales para balancear sus líneas de ensamble [2].

Un artículo más reciente desarrollado por Milas [8] mostró que esta tendencia es aún valida hoy en día. Milas encontró que la mayoría de las compañías llevan a cabo su balanceo de línea basados en precedentes históricos o en la intuición de sus ingenieros.

\section{MODELO MATEMÁTICO DEL SALBP-2}

\section{- Índices}

i: tareas. j: estaciones.

\section{- Parámetros}

$n=$ Número de tareas $(\mathrm{i}=1, \ldots, \mathrm{n})$.

$m=$ Número máximo de estaciones $(\mathrm{j}=1, \ldots, \mathrm{m})$.

$t_{i}=$ Tiempo de procesamiento de una tarea $\mathrm{i}$.

$P D_{i}=$ Conjunto de los predecesores inmediatos de la tarea i.

- Variables de decisión

$$
\begin{aligned}
& x_{i j} \in[0,1] \\
& =\left\{\begin{array}{l}
1 \text { si la tarea } \text { i es asignada a una estación } j \\
0 \text { de lo contrario }
\end{array}\right.
\end{aligned}
$$

$c=$ Tiempo de ciclo.

\section{- Función objetivo}

$\operatorname{Min} Z=c(1)$

\section{- Restricciones}

$$
\begin{aligned}
& \sum_{j=1}^{m} x_{i j}=1 \\
& \sum_{i=1}^{n} t_{i} * x_{i j} \leq c \quad \forall j \\
& \sum_{j=1}^{m} j * x_{p j} \leq \sum_{j=1}^{m} j * x_{i j} \forall i ; \forall p \in P D_{i}
\end{aligned}
$$

La función objetivo consiste en minimizar el tiempo de ciclo, la primera restricción garantiza que todas las tareas i se asignan a una y sólo una estación de trabajo, la segunda restricción garantiza que la suma de los tiempos de procesamiento de las tareas asignadas a la estación $\mathrm{j}$, no excedan el tiempo de ciclo, y la última restricción garantiza el cumplimiento de las relaciones de precedencia.

\section{CASO DE ESTUDIO}

La empresa caso de estudio es una ferretería que cuenta con 3 puntos de venta cada uno en distintas ciudades además de 2 bodegas de almacenaje en dos de estas ciudades que cumplen también su función como centros de distribución. En lo referente a la variedad de productos y artículos, presentan un surtido de aproximadamente 10.000 referencias o ítems, más de 50 distribuciones directas de fábrica y sistema de autoservicio. En las distribuciones directas de fábrica presentan 29 líneas, algunas de ellas son cementos, galvanizados, ladrillos, pintura y cerámica entre otras. En este caso de estudio se parte de los siguientes supuestos: 
- Los días trabajados tienen la misma intensidad horaria.

- Las dos bodegas cuentan con los recursos necesarios de equipo e infraestructura para poder operar.

- El producto final es el pedido.

- Las tareas que se consideran en el subproceso parten de la activación de una orden de cargue, hasta el alistamiento y disposición para el despacho.

- Se definen 3 estaciones de trabajo para las 2 bodegas y cada uno de los modos de venta.

- La valoración del ritmo del trabajo se considera normal (100\%).

- Las actividades que se realizan diariamente y no hacen parte de los procesos en estudio, se llevan a cabo en cualquier momento del transcurso de un día laboral, por lo tanto la unidad de medida está definida como minuto/día

- En pedidos al mayoreo toda ruta carga ítems de todos los sectores establecidos en la bodega.

- En caso de presentarse tiempo libre para un operario después de cumplir con las horas-hombre tanto para el subproceso como a las actividades adicionales que le corresponda en una jornada laboral, este es dedicado para otro tipo de actividades extraordinarias de la bodega que se pueden presentar, que son fortuitas, variables y de compleja medición.

\section{Distribución actual de cargas de trabajo}

A continuación, se muestra la manera como están distribuidas las cargas de trabajo (hora-hombre) según el modo de venta que corresponda y al cumplimiento de las actividades externas asignadas. Para esto, se plantean dos escenarios: distribución de la carga de trabajo por tarea y distribución de la carga de trabajo por operario, en una jornada de trabajo normal de 8 horas diarias.

\begin{tabular}{|c|c|c|c|c|}
\hline $\begin{array}{c}\text { Modo } \\
\text { de } \\
\text { venta }\end{array}$ & Tarea & $\begin{array}{c}\text { Tiempo } \\
\text { requerido } \\
\text { para el } \\
\text { subproceso }\end{array}$ & \begin{tabular}{|c|} 
Tiempo \\
requerido \\
para \\
actividades \\
externas
\end{tabular} & $\begin{array}{c}\text { Tiempo } \\
\text { total } \\
\text { requerido }\end{array}$ \\
\hline \multirow{3}{*}{ Detal } & Orden de cargue & 0,84 & \multirow{3}{*}{0,83} & \multirow{3}{*}{8,37} \\
\hline & Facturación & 5,99 & & \\
\hline & $\begin{array}{l}\text { Disposición para } \\
\text { despacho }\end{array}$ & 0,70 & & \\
\hline $\begin{array}{c}\text { Mayoreo } \\
\text { y detal }\end{array}$ & $\begin{array}{l}\text { Localización- } \\
\text { manipulación y } \\
\text { traslado }\end{array}$ & 36,23 & 18,08 & 54,30 \\
\hline \multirow{3}{*}{ Mayoreo } & Orden de cargue & 1,38 & \multirow{3}{*}{20,33} & \multirow{3}{*}{23,45} \\
\hline & Facturación & 1,20 & & \\
\hline & $\begin{array}{l}\text { Disposición para } \\
\text { despacho }\end{array}$ & 0,54 & & \\
\hline & Total & 46,88 & 39,24 & 86,13 \\
\hline
\end{tabular}

Tabla 1.Distribución actual de la carga de trabajo por tarea en la bodega de la ciudad 1 .

En el primer escenario (Ver Tabla 1), se muestra el tiempo total requerido de horas/hombre para cada modalidad de venta, el cual es el resultado de los tiempos requeridos por cada tarea del subproceso, y el correspondiente a las actividades externas. Mientras que el segundo escenario (Ver tabla 2) se enfoca a la distribución de cargas de trabajo por operario según las tareas asignadas por modalidad de venta y las externas al subproceso, dejando como resultado el tiempo libre o excedido que le corresponde a cada operario según una jornada laboral.

\begin{tabular}{|c|c|c|c|c|}
\hline $\begin{array}{l}\text { Modo de } \\
\text { venta }\end{array}$ & Tarea & \begin{tabular}{|c|} 
Tiempo \\
requerido \\
para el \\
subproceso
\end{tabular} & $\begin{array}{c}\text { Tiempo } \\
\text { requerido } \\
\text { para } \\
\text { actividades } \\
\text { externas }\end{array}$ & $\begin{array}{l}\text { Tiempo total } \\
\text { requerido }\end{array}$ \\
\hline \multirow[t]{2}{*}{ Detal } & $\begin{array}{l}\text { Orden de } \\
\text { cargue }\end{array}$ & 0,62 & \multirow[t]{2}{*}{0,98} & \multirow[t]{2}{*}{9,71} \\
\hline & Facturación & 8,12 & & \\
\hline $\begin{array}{c}\text { Mayoreo y } \\
\text { detal }\end{array}$ & $\begin{array}{l}\text { Disposición } \\
\text { para } \\
\text { despacho }\end{array}$ & 8,21 & 3,68 & 11,88 \\
\hline $\begin{array}{c}\text { Mayoreo y } \\
\text { detal }\end{array}$ & $\begin{array}{l}\text { Localización, } \\
\text { manipulación } \\
\text { y traslado }\end{array}$ & 48,52 & 36,04 & 84,57 \\
\hline \multirow{2}{*}{ Mayoreo } & $\begin{array}{l}\text { Orden de } \\
\text { cargue }\end{array}$ & 1,34 & \multirow{2}{*}{17,23} & \multirow{2}{*}{19,70} \\
\hline & Facturación & 1,12 & & \\
\hline \multicolumn{2}{|c|}{ TOTAL } & 67,93 & 57,93 & 125,86 \\
\hline
\end{tabular}

Tabla 2.Distribución actual de la carga de trabajo por tarea en la bodega de la ciudad 2.

\begin{tabular}{|c|c|c|c|c|c|c|c|}
\hline $\begin{array}{l}\text { Modo de } \\
\text { venta }\end{array}$ & $\begin{array}{c}\text { Operarios } \\
\text { involucrados } \\
\text { en el } \\
\text { subproceso }\end{array}$ & Tareas & $\begin{array}{c}\text { Tiempo } \\
\text { disponi } \\
\text { ble para } \\
\text { jornada } \\
\text { de } \\
\text { trabajo } \\
\text { (horas) }\end{array}$ & $\begin{array}{c}\text { Tiempo } \\
\text { requerido } \\
\text { para el } \\
\text { subproceso }\end{array}$ & $\begin{array}{l}\text { Tiempo } \\
\text { requerid } \\
\text { o para } \\
\text { activida } \\
\text { des } \\
\text { externas }\end{array}$ & $\begin{array}{c}\text { Tiemp } \\
0 \\
\text { labora } \\
\text { do } \\
\text { (horas) }\end{array}$ & $\begin{array}{c}\text { Tiemp } \\
\text { o libre } \\
0 \\
\text { excedi } \\
\text { do } \\
\text { (horas) }\end{array}$ \\
\hline Mayoreo & $\begin{array}{l}\text { Jefe de } \\
\text { bodega }\end{array}$ & \begin{tabular}{|l|} 
Orden de cargue \\
Disposición para \\
despacho
\end{tabular} & 8 & 1,23 & 7,33 & 8,56 & $-0,56$ \\
\hline Mayoreo & $\begin{array}{l}\text { Coordinador } \\
\text { Logístico }\end{array}$ & Orden de cargue & 8 & 0,69 & 10,92 & 11,61 & $-3,61$ \\
\hline $\begin{array}{l}\text { Mayoreo } \\
\text { y Detal }\end{array}$ & Bodeguero & $\begin{array}{l}\text { Localización, } \\
\text { manipulación y } \\
\text { traslado }\end{array}$ & 8 & 7,25 & 3,62 & 10,86 & $-2,86$ \\
\hline Mayoreo & Facturador & Facturación & 4 & 1,20 & 2,08 & 3,29 & 0,71 \\
\hline \multirow{3}{*}{ Detal } & \multirow{3}{*}{ Despachador } & Orden de cargue & \multirow{3}{*}{8} & \multirow{3}{*}{7,53} & \multirow{3}{*}{0,83} & \multirow{3}{*}{8,37} & \multirow{3}{*}{$-0,37$} \\
\hline & & Facturación & & & & & \\
\hline & & $\begin{array}{l}\text { Disposición para } \\
\text { despacho }\end{array}$ & & & & & \\
\hline \multicolumn{4}{|c|}{ Total } & 17,90 & 24,78 & 42,68 & \\
\hline
\end{tabular}

Tabla 3.Distribución actual de la carga de trabajo por operario en la bodega de la ciudad 1.

De acuerdo al resultado actual de la distribución de las cargas de trabajo, se puede observar que diariamente en una jornada normal en la bodega de la ciudad 1 se presenta un tiempo 
excedido de 7,40 horas/hombre de acuerdo al personal mostrado en la Tabla 3, sin embargo cabe destacar que para la tarea de localización manipulación y traslado se cuenta con 5 bodegueros, por ende las 2,86 horas excedidas mostradas en la tabla, corresponden en total a 14,30 horas-hombre, esto quiere decir que el tiempo total excedido en una jornada incluyendo a todo el personal es de 18,84 horas/hombre. Por otro lado, se presenta un total de 0,71 horas/hombre de tiempo libre.

\begin{tabular}{|c|c|c|c|c|c|c|c|}
\hline $\begin{array}{c}\text { Modo de } \\
\text { venta }\end{array}$ & $\begin{array}{c}\text { Operarios } \\
\text { involucrados } \\
\text { en el } \\
\text { subproceso }\end{array}$ & Tareas & \begin{tabular}{|c|} 
Tiempo \\
disponible \\
para \\
jornada de \\
trabajo
\end{tabular} & $\begin{array}{c}\text { Tiempo } \\
\text { requerido } \\
\text { para el } \\
\text { subproceso }\end{array}$ & $\begin{array}{c}\text { Tiempo } \\
\text { requerido } \\
\text { apra } \\
\text { actividades } \\
\text { externas }\end{array}$ & $\begin{array}{r}\text { Tiempo } \\
\text { laborado } \\
\text { (horas) }\end{array}$ & $\begin{array}{l}\text { Tiempo } \\
\text { libre } \\
\text { excedido } \\
\text { (horas) }\end{array}$ \\
\hline \multirow[b]{2}{*}{ Mayoreo } & $\begin{array}{l}\text { Coordinador } \\
\text { logistico }\end{array}$ & $\begin{array}{l}\text { Orden de } \\
\text { cargue }\end{array}$ & 8 & 1,34 & 8,90 & 10,23 & $-2,23$ \\
\hline & Facturador & Facturación & 8 & 1,12 & 8,34 & 9,46 & $-1,46$ \\
\hline \multirow{2}{*}{$\begin{array}{c}\text { Mayoreo y } \\
\text { detal }\end{array}$} & Jefe de bodega & $\begin{array}{l}\text { Disposición } \\
\text { para } \\
\text { despacho } \\
\end{array}$ & 8 & 8,21 & 3,68 & 11,88 & $-3,88$ \\
\hline & Bodeguero & $\begin{array}{l}\text { Localización, } \\
\text { manipulación } \\
\text { y traslado }\end{array}$ & 8 & 4,28 & 7,21 & 11,49 & $-3,49$ \\
\hline \multirow{4}{*}{ Detal } & Bodeguero & $\begin{array}{l}\text { Localización, } \\
\text { manipulación } \\
\text { y traslado }\end{array}$ & 8 & 8,85 & 7,21 & 16,06 & $-8,06$ \\
\hline & Despachador & $\begin{array}{l}\text { Orden de } \\
\text { cargue }\end{array}$ & 8 & 8,74 & 0,98 & 9,71 & $-1,71$ \\
\hline & & Facturación & & & & & \\
\hline & \multicolumn{3}{|c|}{ TOTAL } & 32,54 & 36,32 & 68,83 & \\
\hline
\end{tabular}

Tabla 4.Distribución actual de la carga de trabajo por operario en la bodega de la ciudad 2

Teniendo en cuenta estos resultados, en la distribución actual de las cargas de trabajo se puede determinar que diariamente en una jornada normal en la bodega de la ciudad 2 se presenta un tiempo excedido de 20,84 horas/hombre de acuerdo al personal mostrado en la Tabla 4, sin embargo cabe destacar que para la tarea de localización manipulación y traslado se cuenta con 6 bodegueros, donde uno de ellos se dedica a detal y mayoreo, mientras los 5 restantes se encargan de los pedidos por detal, por lo tanto las 8,06 horas excedidas que se muestran en total corresponden a 40,28 horas-hombre, esto quiere decir que el tiempo total excedido en una jornada incluyendo a todo el personal es de 53,06 horas/hombre. También se puede concluir que para Palmira no se presenta tiempo libre.

\section{ANALISIS Y RESULTADOS}

Este estudio se desarrolla a través de un modelo matemático de programación lineal, el cual es programado por el software AMPL y es resuelto a través del NEOS SOLVER con el solver KNITRO.
El problema actual se trata como un SALBP-II, dónde se fijan 4 estaciones de trabajo. Con base a las horas-hombre requeridas y la distribución actual del recurso humano descritos en el capítulo anterior, se puede determinar que en las 2 bodegas existe una carga de trabajo excedida para cada una de las tareas del subproceso en una jornada laboral, donde el mayor exceso se encuentra en la tarea de localización, manipulación y traslado, la cual involucra al bodeguero y es la concerniente al proceso de picking (Ver Tablas 5 y 6 ).

El mayor exceso de carga de trabajo se encuentra en la tarea que involucra a los bodegueros, por tanto la estrategia estará en torno a la utilización de la técnica de balanceo de línea que concierne a la reducción del tiempo de ciclo, esto proporcionará una disminución en el tiempo estándar y a su vez en la carga de trabajo, específicamente para la tarea de localización, manipulación y traslado; afectando de manera positiva la carga total de la jornada laboral de cada una de las bodegas obteniendo los siguientes resultados.

\section{Para la ciudad 1}

\begin{tabular}{|c|c|c|c|c|c|}
\hline $\mathrm{N}^{\circ}$ & Tarea & T.E (min) & $\begin{array}{c}\text { Tiempo } \\
\text { total } \\
\text { requerido } \\
\text { (min) }\end{array}$ & $\begin{array}{c}\text { Tiempo total } \\
\text { requerido } \\
\text { (horas/hombre) }\end{array}$ & $\begin{array}{c}\text { Carga de } \\
\text { trabajo por } \\
\text { estación } \\
\text { hora/hombre) }\end{array}$ \\
\hline 1 & Orden de cargue & 0,35 & 50,69 & 0,84 & 0,84 \\
\hline 2 & $\begin{array}{l}\text { Localización, } \\
\text { manipulación y } \\
\text { traslado }\end{array}$ & 6,69 & 977,08 & 16,28 & 16,28 \\
\hline 3 & Facturación & 2,46 & 359,11 & 5,99 & \multirow[b]{2}{*}{6,69} \\
\hline 4 & $\begin{array}{l}\text { Disposición para } \\
\text { el despacho }\end{array}$ & 0,29 & 42,27 & 0,7 & \\
\hline & TOTAL & 9,79 & 1429,15 & 23,81 & 23,81 \\
\hline
\end{tabular}

Tabla 5.Subproceso de detal intervenido en la tarea 2 para la bodega de la ciudad 1 .

\begin{tabular}{|c|c|c|c|c|c|}
\hline No & Tarea & $\underset{\text { (min) }}{\mathrm{T} . \mathrm{E}}$ & $\begin{array}{c}\text { tiempo } \\
\text { total } \\
\text { requerido } \\
\text { (min) }\end{array}$ & $\begin{array}{c}\text { tiempo total } \\
\text { requerido } \\
\text { (horas/hombr } \\
\text { e) }\end{array}$ & \begin{tabular}{|c|}
$\begin{array}{c}\text { Carga de } \\
\text { trabajo por } \\
\text { estación } \\
\text { (hora/hombr } \\
\text { e) }\end{array}$ \\
\end{tabular} \\
\hline 1 & Orden de cargue & 0,32 & 36,95 & 0,62 & 0,62 \\
\hline 2 & $\begin{array}{l}\text { Localización, } \\
\text { manipulación y } \\
\text { traslado }\end{array}$ & 12,07 & 1375,48 & 22,92 & 22,92 \\
\hline 3 & Facturación & 4,27 & 487,20 & 8,12 & \multirow[b]{2}{*}{15,96} \\
\hline 4 & $\begin{array}{l}\text { Disposición para el } \\
\text { despacho }\end{array}$ & 5,50 & 470,23 & 7,84 & \\
\hline & $\Sigma$ & 22,16 & 2369,87 & 39,50 & 39,50 \\
\hline
\end{tabular}

Tabla 6.Subproceso detal intervenido en la tarea 2 para la bodega de la ciudad 2.

Con respecto al tiempo de entrega de los productos, se tiene que es medido por el tiempo de ciclo del pedido, en este caso se 
mide a partir de los centros de distribución y debido a que se tendrá un nivel de servicio efectivo, se asume que no habrá stock outs, es decir, que los centros de distribución siempre tendrán a disposición el tipo y cantidad de producto que el cliente requiera. A continuación se muestra el tiempo de ciclo del pedido en la Tabla 7.

\begin{tabular}{|c|c|}
\hline $\begin{array}{c}\text { NÚMERO } \\
\text { DE CEDIS } \\
\text { ABIERTOS }\end{array}$ & $\begin{array}{c}\text { TIEMPO } \\
\text { TOTAL CICLO } \\
\text { DEL PEDIDO } \\
\text { (Horas) }\end{array}$ \\
\hline 1 & 166,15 \\
\hline 2 & 147,95 \\
\hline 3 & 76,29 \\
\hline 4 & 66,66 \\
\hline 5 & 63,8 \\
\hline 6 & 59,19 \\
\hline 7 & 55,85 \\
\hline 8 & 55,85 \\
\hline 9 & 55,85 \\
\hline
\end{tabular}

Tabla 7.Tiempo de ciclo de pedido a partir de la localización de centros de distribución. Fuente: elaboración propia.

El modelo de localización que se aplicó para esta empresa caso de estudio, arroja como resultado la minimización de costos totales logísticos con 4 Cedis. Haciendo el cálculo del tiempo de ciclo para este número de cedis resulta en 66,66 horas como se muestra en la Tabla 7

\section{CONCLUSIONES}

Los diferentes métodos y técnicas desarrolladas para la resolución de los ALPB son aplicables a cualquier tipo de problema que pretenda lograr un equilibrio de cargas de trabajo. El estudio desarrollado mostró que a través del modelo matemático de programación lineal propuesto por Baybars se logró establecer el tiempo de ciclo actual del subproceso de movimiento y despacho de pedidos, así como la asignación de las tareas que hacen parte de este, a cada una de las estaciones. Cabe resaltar que, debido a la simplicidad del subproceso en estudio, este modelo matemático para los SALBP-2 no refleja su verdadera utilidad dado que presenta resultados casi obvios, sin embargo, éste puede ser aplicable a cualquier situación y problemas de mayor complejidad.

Por otro lado, a través del análisis del caso de estudio se corroboró que la tarea involucrada con el picking es la que demanda más tiempo y requiere de una mayor intensidad de mano de obra, razones que la convierten en el cuello de botella de todo el subproceso. De acuerdo a esto, una forma de mejorar la eficiencia del subproceso es atacando las operaciones inmersas en esta tarea, a través de estrategias que permitan encontrar un equilibrio en las cargas de trabajo de cada estación como por ejemplo la asignación de estaciones en paralelo con la misma cantidad de recursos, lo que al final impactará en el requerimiento total de horas/hombre.

\section{REFERENCIAS}

[1]. Barrios, M. (2010). Balanceo de líneas (análisis de la producción). Disponible en: http://es.scribd.com/.

[2]. Chase, R.B. (1974).Survey of paced assembly systems, Industrial Engineering, 6.82-90.

[3]. Díaz, F. J. González, M. (2011) Estado del arte en el problema de balanceo de líneas de ensamble. En: Academia Journals. Congreso Internacional de Investigación. Volumen 3, No. 2.

[4]. Espinosa, F. Apuntes sobre métodos y tiempos. En línea: < http://ing.utalca.cl/ fespinos/22Metodos_tiempos.pdf $>$. Disponible en: Universidad de Talca: Facultad de ingeniería.

[5]. Gutjahr, A.L. and Nemhauser, G.L. (1964) An algorithm for the balancing problem, Management Science, 11, 23-35.

[6]. Heizer, J. y Render, B. (2004) Balanceo de la línea de ensamble. Principios de administración de operaciones. (5 Ed). México: Pearson Educación, 351.

[7]. Meza, G. Bedoya, J. A. (2009) Optimización en procesos y asignación de recursos en las empresas del sector confección a través del balanceo de líneas de producción. Universidad cooperativa de Colombia, sede Pereira. 18, 66-69.

[8]. Milas, G. (1990) Assembly line balancing: Let's remove the mystery, Industrial Engineering, 22 12-18.

[9]. Restrepo, J. H. Medina, V. P. D. Cruz T. E. A. (2006) Balanceo de un módulo de confección utilizando el algoritmo de Helgeson and Birnie: Un caso de estudio. 
Scientia et technica.UTP. ISSN 0122-1701. Universidad Tecnológica de Pereira.

[10]. Seyed, A.Fatemighomi, M. Zandieh.A. (2011) simulated annealing algorithm for balancing the assembly line type II problem with sequencedependent setup times between tasks. En: International Journal of Production Research, Vol. 49, No. 3. 